starkem Weingeist lösliche Chlorophyll in sich aufzunehmen.

Dars natürlich 5) das Verdampfen des Extracts im Wasserbade, wie die Vorschrift befiehlt, geschehen mufs, braucht eigentlich nicht erwähnt zu werden, gar oft wird aber auch dieses versäumt, and dann natürlich auch lann leicht eine Zersetzung des Chlorophylls eintreten.

In solcher Weise glaube ich die Ursache der Verschiedenheit der erwähnten Extracte hinlänglich erklärt zu haben. Wahrscheinlich macht dieser grüne Pflanzenstoff nur einen untergeordneten Theil der Wirksamlieit der Extracte überhaupt ans, scine Menge beträgt immer nur wenig (mag nach Verschiedenheit der Gattung auf $\frac{1}{2}$ Pfund Extract nur 1 bis 4 Quentch. betragen), so dafs auch ein nicht besonders grün erscheinendes (nur nicht braunes) Extract gewifs noch ein wirksames Mittel sein wird, denn alle narkotischen Extracte sind nur als Mischungen von ei renthümlichen Pflanzensalzen (Verbindungen des Alkaloids mit Pflanzensänre) mit Extractivstoff; Chlorophyll etc. zu betrachten. Ein schön grünes Extract des Aconits, der Belladonna, der Digitalis, des Coniums und Hyoscyamus (bei letzteren beiden entscheidet auch der (Geruch) etc., ist übrigens immer ein beweis der Sorgfalt seines Verfertigers und ein Anhaltpunct für seine richtige und vorschriftsmälsige Bereitung.

\title{
Notiz über die Bereitung der narkotischen Extracte;
}

vom

Hofapotheker Dr. Bucholz in Gotha.

Zu dem vorstehenden Aufsatze meines Freundes Jahn über die narkotischen Extracte erlaube ich mir nachfolgende kleine Bemerkungen.

Es lömmt vorzüglich darauf an, nach Zusatz der chlorophyllhaltigen Flüssigkeit, nachdem der Weingeist abdestillirt, zur ersten dicklichen Extractmasse, das Ganze anhaltend zu agitiren, da ohne diese Beachtung das Chlorophyll nicht fein genug zertheilt wird, und 
dem Extracte auch nicht die schöne Färbung geben kann.

Ich habe ferner für gut gefunden, dafs das Abdampfen der narkotischen Extracte im Wasserbade bis zur vorschriftsmälsigen Consistenz für die möglichste Güte des Extracts noch nicht genügt. Die Wärme eines solchen Wasserbades, die doch zu $7^{\circ}$ R. und darüber steigt, trägt gewifs zur wenigen guten Beschaffenheit und Wirkung des narkotischen Extractes bei. Nachdem die Extractmasse dünne Mellago-Consistenz erreicht hat, lasse ich dieselbe daher in eine in den $\mathrm{Ab}$ dampflessel gestellte Porcellanschale bringen, wo die Temperatur 50 bis $60^{\circ} \mathrm{h}$. hält, und so unter stetem Umrühren die Abdampfung zu Ende bringen *).

Fünfte Abtheilung.

\section{Arzneimittelpriffung.}

\section{Opiumprobe für eine chemische Fabrik; von}

\section{Thiboumery.}

1 Kilogramm fein zerschnittencs Opium läfst man viermal hintereinander jedesmal mit einem Liter Wasser

*) Die vorstehenden Beobachtungen der Herren $J$ a h $n$ und Bucholz stimmen mit meinen Erfahrungen völlig überein, und jeder aufmerksame Beobachter wird sie richtig finden. Soll das Extract eine grüne Farbe behalten, so kömmt es sehr auf das innige Vermengen desselben mit dem in körnigen und häutigen Stücken ausgeschiedenen Chlorophyll an. Uebrigens lege ich auf das Vorhandensein dieses Stoff́s in den warkotischen Extracten keinen Werth, ich finde es passender, denselben daraus abzuscheiden, da, nach meinem Dafürhalten, die Wesenheit der narkotischen Extracte, die ich stets fur eine sehr wichtige Klasse von Arzneimitteln halte, in den organischen Verbindungen der Alkaloide mit den Pflanzensäuren und in einigen Fällen mit einigen andern eigenthiimlichen Stoffen besteht, wie auch Herr Jahn anfihrt, und ich ebenfalls bereits vor siebzehn Jahren seigte. Vergl. diese Zeitschrift 1. Reihe I. Bd. S. 184.

Br. 